\title{
Expression of the interferon regulatory factor 6 in non-small cell lung carcinoma
} distinguishes squamous cell lung carcinoma from adenocarcinoma.

Shahan Mamoor, MS1

1'shahanmamoor@gmail.com

East Islip, NY USA

Non-small cell lung cancer, classified as adenocarcinomas or squamous cell lung carcinomas, is the major cause of cancer death in the United States and worldwide ${ }^{1}$. To understand the most significant transcriptional differences between adenocarcinomas and squamous cell lung carcinomas, we mined published microarray data from two separate studies ${ }^{2,3}$. We identified interferon regulatory factor 6 (IRF6) as a distinguishing transcriptional feature of squamous cell lung carcinomas, suggesting the biology of IRF6 may be relevant to the pathways critical for the development or maintenance of squamous cell lung carcinomas but not of adenocarcinomas. IRF6 expression was significantly correlated with prognosis of patients with NSCLC, as patients with low tumor expression of IRF6 possessed significantly longer median overall survival than those with high tumor expression of IRF6. These analyses will also provide novel tools for diagnostic approaches and for guidance of treatment regimens for a cancer with dismal outlook.

Keywords: interferon regulatory factor 6, IRF6, non-small cell lung cancer, adenocarcinoma, squamous cell lung carcinoma, systems biology of NSCLC, targeted therapeutics in NSCLC, targeted diagnostics in NSCLC. 
Non-small cell lung cancer is the single most common cancer in the United States and the most common cause of cancer death in the United States ${ }^{1}$. The two major types of nonsmall cell lung cancer are adenocarcinoma and squamous cell lung carcinoma ${ }^{4}$.

Treatment approaches and prognosis can differ based on whether a patient is diagnosed with adenocarcinoma or squamous cell lung carcinoma ${ }^{4}$, and current approaches to distinguishing adenocarcinoma from squamous cell lung carcinoma rely on time-consuming procedures involving immunohistochemical staining with multiple markers including TTF1, p40, p63, CK5 and/or CK7 and subsequent readout by pathologists who will proceed to label each section, in a binary fashion, as positive or negative for each marker ${ }^{5}$. To understand the molecular nature of ACC and SCLC tumors in an unbiased fashion and the systems level, and to facilitate discovery of genes with utility in NSCLC diagnostics in a quantitative real-time workflow providing a quantitative and independent measure rather than qualitative measure based on pathologist interpretation, we performed comparative transcriptome analysis ${ }^{2,3}$ of the two major types of NSCLC tumors: adenocarcinomas and squamous cell lung carcinomas.

This blind, systems-level approach identified the gene encoding interferon regulatory factor 6, IRF6, as among the genes most differentially expressed when comparing adenocarcinomas and squamous cell lung carcinoma tumors, with IRF6 expression distinguishing squamous cell lung carcinomas from adenocarcinomas, suggesting IRF6 could be a useful tool in NSCLC diagnostics.

\section{Methods}

We utilized datasets GSE $74706^{2}$ and GSE $33532^{3}$ for this comparative transcriptome analysis of adenocarcinomas and squamous cell lung carcinomas. GSE74706 was generated using Agilent-026652 Whole Human Genome Microarray 4x44K v2 technology; for this analysis, we used $n=10$ adenocarcinomas and $n=4$ squamous cell lung carcinomas, and the analysis was performed using platform GPL13497. GSE33532 was generated using Affymetrix Human Genome U133 Plus 2.0 Array technology; for this analysis, we used $n=10$ adenocarcinoma tumors and $n=8$ squamous cell lung carcinomas, and the analysis was performed using platform GPL570.

The Benjamini and Hochberg method of $p$-value adjustment was used for ranking of differential expression but raw $p$-values were used to assess statistical significance of global differential expression. Log-transformation of data was auto-detected, and the NCBI generated category of platform annotation was used. A statistical test was performed to evaluate whether IRF6 expression was significantly between adenocarcinoma and squamous cell carcinoma using a two-tailed, unpaired t-test with Welch's correction. We used PRISM for all statistical analyses of differential gene expression in NSCLC tumors (Version 8.4.0)(455). For Kaplan-Meier survival analysis, we used the Kaplan-Meier plotter online tool ${ }^{7}$ for correlation of IRF6 mRNA expression levels with overall survival in $n=1925$ non-small cell lung cancer patients. 


\section{Results}

We mined published microarray data from two separate studies ${ }^{2,3}$ to describe in an unbiased fashion and at the systems-level genes whose expression was most specific to either one of the two major types of non-small cell lung cancer: adenocarcinomas and squamous cell lung carcinomas.

\section{IRF6 expression distinguishes squamous cell lung carcinomas from adenocarcinomas.}

By comparing the global gene expression profiles of squamous cell lung carcinomas to adenocarcinomas, we found that the interferon regulatory factor 6 was among the most differentially expressed genes between the two major sub-types of NSCLC2 (Table 1). When sorting each of the genes expression by microarray based on significance of change in expression between adenocarcinomas and squamous cell lung carcinomas, IRF6 ranked 86 out of 34183 total transcripts (Table 1). IRF6 differential expression between adenocarcinomas and squamous cell lung carcinomas was statistically significant (Table $1 ; p=2.6 \mathrm{E}-06$ ).

In a separate dataset ${ }^{3}$, IRF6 was again amongst the most differentially expressed when comparing squamous cell lung carcinomas to adenocarcinomas in NSCLC (Table 2). When sorting each of the genes expression by microarray based on significance of change in expression between adenocarcinomas and squamous cell lung carcinomas, IRF6 ranked 65 out of 25906 total transcripts (Table 2). IRF6 differential expression between adenocarcinomas and squamous cell lung carcinomas was again statistically significant (Table $2 ; p=2.39 \mathrm{E}-18$ ).

\section{Interferon regulatory factor 6 is expressed at significantly higher levels in squamous cell} lung carcinomas than in adenocarcinomas.

We obtained exact mRNA values for IRF6 to understand the magnitude and direction of difference in IRF6 mRNA expression between adenocarcinomas and squamous cell lung carcinomas in NSCLC. IRF6 was expressed at significantly higher levels in squamous cell lung carcinomas as compared to adenocarcinomas, and this difference was statistically significant (Figure 1 and Figure 2: $p<0.0001$ and $p=0.0005$, respectively). We calculated a mean fold change of $1.5362 \pm 0.1160$ in IRF6 expression when comparing squamous cell lung carcinomas to adenocarcinomas (Table 2).

\section{Expression of IRF6 is associated with patient survival in NSCLC.}

We performed Kaplan Meier survival analysis ${ }^{7}$ to determine if IRF6 expression was correlated with patient outcomes in NSCLC. We found significant correlation between expression of IRF6 and overall survival in patients with NSCLC (Figure 3 and Table 3); high IRF6 tumor expression was a negative prognostic indicator. While median overall survival was 98.5 months for NSCLC patients with low expression of IRF6, median overall survival was 52 months for NSCLC patients with high expression of IRF6. Correlation of IRF6 tumor expression with median OS with statistically significant (Figure 3; log rank p-value: 3.9e-06; hazard ratio: $1.47(1.25-1.74))$. 
Thus, by mining published microarray data from independent datasets, we found that IRF6 was among the genes whose expression most significantly distinguished squamous cell lung carcinomas from adenocarcinomas in NSCLC. Moreover, we found a statistically significant correlation between IRF6 tumor expression at the mRNA level and patient survival in NSCLC.

\section{Discussion}

To understand the most striking transcriptional features of each of the two major tumor types in NSCLC, we performed comparative transcriptome analysis of ACC and SCLC tumors using published microarray data, discovering interferon regulatory factor 6 as the among the most distinguishing transcriptional feature of squamous cell lung carcinomas.

IRF6 belongs to the family of interferon regulatory transcription factors that translocate to the nucleus and occupy interferon response regulatory elements in genomic DNA to transactivate expression of interferon-inducible genes following signaling downstream of infectious triggers, most typically viral infections, pathogen-associated molecular pattern (PAMP)-associated, or other stimuli8. IRF6 is translated from a message comprised of 10 exons and contains a winged-helix DNA-binding domain, an Smad-interferon regulatory factor-binding domain, or SMIR, which functions in protein binding9. Mice deficient in IRF1, IRF2, IRF3, IRF4, IRF6, IRF7, IRF8 and IRF9 are manifest no developmental phenotypes but all possess immune signaling-related deficits ${ }^{10-16}$. IRF6-deficient mice are the only mice with targeted deletion of an interferon regulatory factor manifesting development phenotypes - abnormalities in the skin, in the limbs and the craniofacial structure ${ }^{17}$. While the skin of wild-type embryonic day 17.5 (E17.5) embryos displayed wrinkling, this was remarkably absent from the skin of IRF6-deficient E17.5 embryos. Electron microscopy of wild-type skin displayed keratohyalin granules and a cornified outer layer, but this was absent in Irf6-deficient embryos. Toluidine blue is excluded from mature skin, as it does not penetrate cornified layers; toluidine blue was excluded from wild-type embryos but not from Irf6-deficient embryos. Proliferation and failure of differentiation of keratinocytes was found to be the source of an expanded spinous layer: while BrdU could be incorporated in both the basal and suprabasal layers of the epidermis in IRF6-deficient embryos ${ }^{17}$, BrdU was incorporated only in the basal layers of wild-type embryos; in addition, TUNEL staining was observed in epidermis from wild-type embryos but not from IRF6-deficient embryos ${ }^{17}$. Global gene expression analysis revealed that loricrin and fillagrin were significantly decreased in granular and cornfield layers, and in the spinous layer, mice deficient in Irf6 could not silence keratin 14 and p6317. In terms of limb defects, the forelimbs of Irf6-null mice were shortened and lacked visible digits, with a caudal projection that lacked visible hindlimb and tail. While Irf6 was not expressed in the bone, the vertebrae, sternum, xiphoid process, digits and skull were all abnormal with a secondary palate cleft ${ }^{17}$. Thus, Irf6 is critical for development of the skin, the limbs, the broader skeletal system and the palate ${ }^{17}$. Accordingly, mutations in the IRF6 gene are the cause of Van der Woude syndrome, an autosomal dominant cleft lip and palate with lip pits, and IRF6 mutations are also found in patients with Popliteal pterygium syndrome, a disorder resulting in orofacial defects, as well as abnormalities of the skin and the genitals ${ }^{18}$. Interactions between the transcription factor p63 and IRF6 are important for cleft palate development in mice ${ }^{19}$; other pathways that intersect with p63 and If in development, specifically in the development of the facial structure through support of epithelial cell death through apoptosis are $\mathrm{Pbx}$ and $\mathrm{Wnt}^{20}$. A function for Irf6 in keratinocyte proliferation, as 
evidenced in mice deficient for Irf6, is supported hyper proliferative epidermis and soft tissue fusion in mice modeling missense mutations found in patients with Van der Woude syndrome, indicating Irf6 is important for the transition from proliferation to differentiation of keratinocytes; Irf6 genetically interacts with 14-3-3 $\sigma$ in this context 21 . In the context of cancer, during epidermal development. Irf6 is a Notch target in keratinocytes, and down-regulation of Irf6 expression supports tumor formation in mice induced by Ras, antagonizing keratinocyte differentiation in vivo ${ }^{22}$. In squamous cell carcinomas, the expression of Irf6 is significantly down-regulated, CpG methylation of the Irf6 promoter correlates with decreased expression of Irf6, and decreased expression of Irf6 correlates with tumor invasiveness and differentiation ${ }^{23}$. Genome-wide Irf6 occupancy analysis demonstrated that Irf6 could directly target genes involved cell cycle, cell adhesion and cell-cell contact 23 . Thus, Irf6 is the only interferon regulatory factor with functions in embryonic development, it is essential for development of the skin, palate and skeletal structure, in humans, mutations in Irf6 result in palate and orofacial defects; Irf6 can antagonize keratinocyte differentiation, its expression can be regulated by p63 and Notch, and Irf6 down-regulation and promoter meythyukationi has been described in squamous cell carcinomas.

We found that the interferon regulatory factor 6 was the among the most differentially expressed gene when comparing the tumors of patients with the two most common sub-types of NSCLC: adenocarcinomas and squamous cell lung carcinomas. We also found significant correlation between overall survival of NSCLC patients and IRF6 expression. IRF6 has value as a diagnostic tool, as a prognostic indicator, and the biology of IRF6 may be of value in understanding fundamental differences between the two major types of the most common type of cancer, and the most common cause of cancer death in the United States and worldwide. 


\section{References}

1. Siegel, R.L., Miller, K.D. and Jemal, A., 2019. Cancer statistics, 2019. CA: a cancer journal for clinicians, 69(1), pp.7-34.

2. Marwitz, S., Depner, S., Dvornikov, D., Merkle, R., Szczygieł, M., Müller-Decker, K., Lucarelli, P., Wäsch, M., Mairbäurl, H., Rabe, K.F. and Kugler, C., 2016. Downregulation of the TGF $\beta$ pseudoreceptor BAMBI in non-small cell lung cancer enhances TGF $\beta$ signaling and invasion. Cancer research, 76(13), pp.3785-3801.

3. Kabbout, M., Garcia, M.M., Fujimoto, J., Liu, D.D., Woods, D., Chow, C.W., Mendoza, G., Momin, A.A., James, B.P., Solis, L. and Behrens, C., 2013. Ets2 mediated tumor suppressive function and met oncogene inhibition in human non-small cell lung cancer. Clinical cancer research, 19(13), pp.3383-3395.

4. Cetin, K., Ettinger, D.S., Hei, Y.J. and D O'Malley, C., 2011. Survival by histologic subtype in stage IV nonsmall cell lung cancer based on data from the Surveillance, Epidemiology and End Results Program. Clinical epidemiology, 3, p.139.

5. Yuan, M., Huang, L.L., Chen, J.H., Wu, J. and Xu, Q., 2019. The emerging treatment landscape of targeted therapy in non-small-cell lung cancer. Signal Transduction and Targeted Therapy, 4(1), pp.1-14.

6. Osmani, L., Askin, F., Gabrielson, E. and Li, Q.K., 2018, October. Current WHO guidelines and the critical role of immunohistochemical markers in the subclassification of non-small cell lung carcinoma (NSCLC): Moving from targeted therapy to immunotherapy. In Seminars in cancer biology (Vol. 52, pp. 103-109). Academic Press.

7. Gyorffy, B., Surowiak, P., Budczies, J. and Lanczky, A., 2013. Online survival analysis software to assess the prognostic value of biomarkers using transcriptomic data in non-smallcell lung cancer. PloS one, 8(12), pp.e82241-e82241.

8. Yanai, H., Negishi, H. and Taniguchi, T., 2012. The IRF family of transcription factors: Inception, impact and implications in oncogenesis. Oncoimmunology, 1(8), pp.1376-1386.

9. Kondo, S., Schutte, B.C., Richardson, R.J., Bjork, B.C., Knight, A.S., Watanabe, Y., Howard, E., de Lima, R.L.F., Daack-Hirsch, S., Sander, A. and McDonald-McGinn, D.M., 2002. Mutations in IRF6 cause Van der Woude and popliteal pterygium syndromes. Nature genetics, 32(2), pp.285-289.

10.Ingraham, C.R., Kinoshita, A., Kondo, S., Yang, B., Sajan, S., Trout, K.J., Malik, M.I., Dunnwald, M., Goudy, S.L., Lovett, M. and Murray, J.C., 2006. Abnormal skin, limb and craniofacial morphogenesis in mice deficient for interferon regulatory factor 6 (Irf6). Nature genetics, 38(11), pp.1335-1340. 
11.Matsuyama, T., Kimura, T., Kitagawa, M., Pfeffer, K., Kawakami, T., Watanabe, N., Kündig, T.M., Amakawa, R., Kishihara, K., Wakeham, A. and Potter, J., 1993. Targeted disruption of IRF-1 or IRF-2 results in abnormal type I IFN gene induction and aberrant lymphocyte development. Cell, 75(1), pp.83-97.

12.Sato, M., Suemori, H., Hata, N., Asagiri, M., Ogasawara, K., Nakao, K., Nakaya, T., Katsuki, M., Noguchi, S., Tanaka, N. and Taniguchi, T., 2000. Distinct and essential roles of transcription factors IRF-3 and IRF-7 in response to viruses for IFN- $\alpha / \beta$ gene induction. Immunity, 13(4), pp.539-548.

13.Mittrücker, H.W., Matsuyama, T., Grossman, A., Kündig, T.M., Potter, J., Shahinian, A., Wakeham, A., Patterson, B., Ohashi, P.S. and Mak, T.W., 1997. Requirement for the transcription factor LSIRF/IRF4 for mature B and T lymphocyte function. Science, 275(5299), pp.540-543.

14.Takaoka, A., Yanai, H., Kondo, S., Duncan, G., Negishi, H., Mizutani, T., Kano, S.I., Honda, K., Ohba, Y., Mak, T.W. and Taniguchi, T., 2005. Integral role of IRF-5 in the gene induction programme activated by Toll-like receptors. Nature, 434(7030), pp.243-249.

15.Holtschke, T., Löhler, J., Kanno, Y., Fehr, T., Giese, N., Rosenbauer, F., Lou, J., Knobeloch, K.P., Gabriele, L., Waring, J.F. and Bachmann, M.F., 1996. Immunodeficiency and chronic myelogenous leukemia-like syndrome in mice with a targeted mutation of the ICSBP gene. Cell, 87(2), pp.307-317.

16.Kimura, T., Kadokawa, Y., Harada, H., Matsumoto, M., Sato, M., Kashiwazaki, Y., Tarutani, M., Sok-Pin Tan, R., Takasugi, T., Matsuyama, T. and Mak, T.W., 1996. Essential and nonredundant roles of p48 (ISGF3y) and IRF-1 in both type I and type II interferon responses, as revealed by gene targeting studies. Genes to Cells, 1(1), pp.115-124.

17.Ingraham, C.R., Kinoshita, A., Kondo, S., Yang, B., Sajan, S., Trout, K.J., Malik, M.I., Dunnwald, M., Goudy, S.L., Lovett, M. and Murray, J.C., 2006. Abnormal skin, limb and craniofacial morphogenesis in mice deficient for interferon regulatory factor 6 (Irf6). Nature genetics, 38(11), pp.1335-1340.

18.Kondo, S., Schutte, B.C., Richardson, R.J., Bjork, B.C., Knight, A.S., Watanabe, Y., Howard, E., de Lima, R.L.F., Daack-Hirsch, S., Sander, A. and McDonald-McGinn, D.M., 2002. Mutations in IRF6 cause Van der Woude and popliteal pterygium syndromes. Nature genetics, 32(2), pp.285-289.

19.Thomason, H.A., Zhou, H., Kouwenhoven, E.N., Dotto, G.P., Restivo, G., Nguyen, B.C., Little, H., Dixon, M.J., van Bokhoven, H. and Dixon, J., 2010. Cooperation between the transcription factors p63 and IRF6 is essential to prevent cleft palate in mice. The Journal of clinical investigation, 120(5), pp.1561-1569. 
20.Ferretti, E., Li, B., Zewdu, R., Wells, V., Hebert, J.M., Karner, C., Anderson, M.J., Williams, T., Dixon, J., Dixon, M.J. and Depew, M.J., 2011. A conserved Pbx-Wnt-p63-Irf6 regulatory module controls face morphogenesis by promoting epithelial apoptosis. Developmental cell, 21(4), pp.627-641.

21.Richardson, R.J., Dixon, J., Malhotra, S., Hardman, M.J., Knowles, L., Boot-Handford, R.P., Shore, P., Whitmarsh, A. and Dixon, M.J., 2006. Irf6 is a key determinant of the keratinocyte proliferation-differentiation switch. Nature genetics, 38(11), pp.1329-1334.

22.Restivo, G., Nguyen, B.C., Dziunycz, P., Ristorcelli, E., Ryan, R.J., Özuysal, Ö.Y., Di Piazza, M., Radtke, F., Dixon, M.J., Hofbauer, G.F. and Lefort, K., 2011. IRF6 is a mediator of Notch pro-differentiation and tumour suppressive function in keratinocytes. The EMBO journal, 30(22), pp.4571-4585.

23.Botti, E., Spallone, G., Moretti, F., Marinari, B., Pinetti, V., Galanti, S., De Meo, P.D.O., De Nicola, F., Ganci, F., Castrignanò, T. and Pesole, G., 2011. Developmental factor IRF6 exhibits tumor suppressor activity in squamous cell carcinomas. Proceedings of the National Academy of Sciences, 108(33), pp.13710-13715. 


\begin{tabular}{|r|l|l|l|l|l|}
\hline Rank & ID & p-value & t & B & Gene \\
\hline 86 & A_23_P748 & $2.6 E-06$ & 6.5990767 & 4.89926 & IRF6 \\
\hline
\end{tabular}

Table 1: Interferon regulatory factor 6 is among the most differentially expressed genes when comparing whole transcriptomes of adenocarcinomas and squamous cell lung carcinomas, the two major types of non-small cell lung cancer.

Rank of differential expression, probe ID, $p$-value with respect to differential expression, $\mathrm{t}$, a moderated t-statistic, $\mathrm{B}$, the log-odds of differential expression between the two groups compared, and gene are listed in this chart. 
Table 2: Interferon regulatory factor 6 is among the most differentially expressed genes when comparing whole transcriptomes of adenocarcinomas and squamous cell lung carcinomas, the two major types of non-small cell lung cancer.

Rank of differential expression, probe ID, $p$-value with respect to differential expression, $\mathrm{t}$, a moderated $\mathrm{t}$-statistic, $\mathrm{B}$, the log-odds of differential expression between the two groups compared, fold change of IRF6 when comparing squamous cell lung carcinomas to adenocarcinomas, gene and gene name are listed in this chart. 


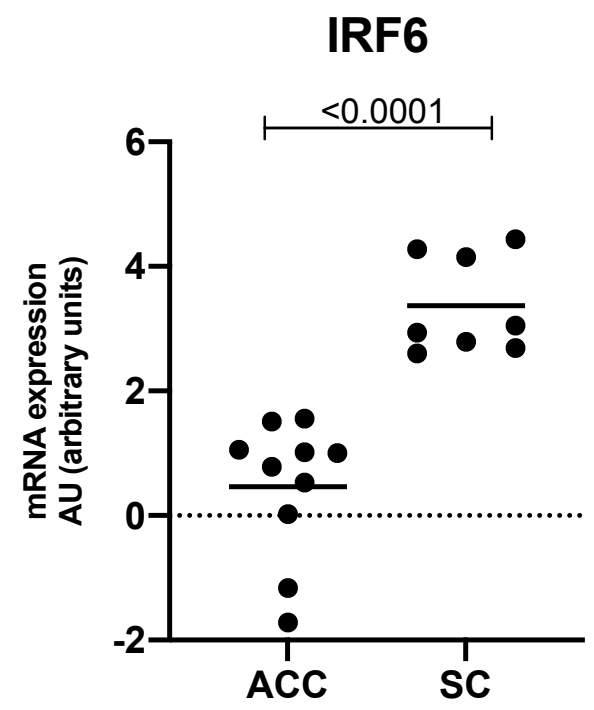

Figure 1: Expression of interferon regulatory factor 6 distinguishes squamous cell lung carcinoma from adenocarcinoma.

Messenger RNA (mRNA) levels of IRF6 in the tumors of patients with adenocarcinoma ("ACC"; left) and in the tumors of patients with squamous cell lung carcinoma ("SC"; right) are graphically represented here with mean mRNA levels marked and the result of a statistical test evaluating significance of difference in mRNA expression between the tumors of patients with adenocarcinomas and squamous cell lung carcinomas, a $p$ value, listed above. 


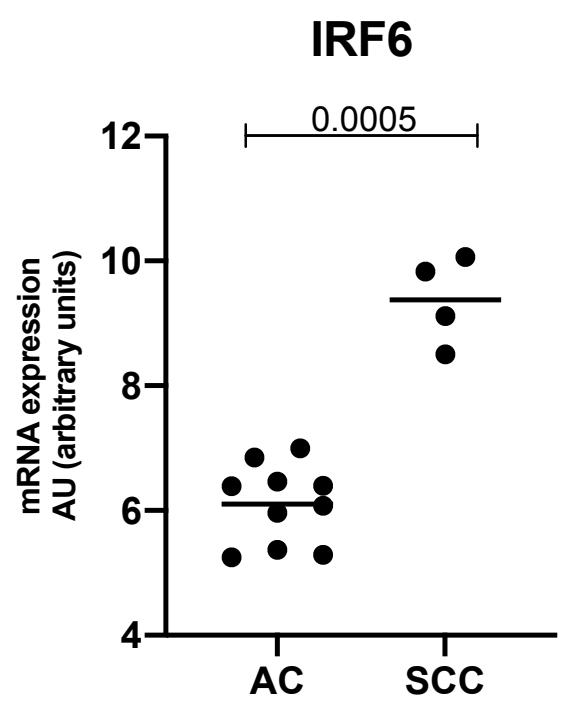

Figure 2: Expression of interferon regulatory factor 6 distinguishes squamous cell lung carcinoma from adenocarcinoma.

Messenger RNA (mRNA) levels of IRF6 the tumors of patients with adenocarcinoma ("ACC"; left) and in the tumors of patients with squamous cell lung carcinoma ("SC"; right) are graphically represented here with mean mRNA levels marked and the result of a statistical test evaluating significance of difference in mRNA expression between the tumors of patients with adenocarcinomas and squamous cell lung carcinomas, a $p$ value, listed above. 
Figure 3: Interferon regulatory factor 6 expression in the tumors of patients with NSCLC correlates with overall survival.

Depicted in this Kaplan-Meier plot is the probability of overall survival for $n=1925$ total patients stratified into two groups, based on low or high expression of IRF6 in patient tumors. The log rank $p$-value denoting statistical significance of difference in overall survival when comparing the two groups, as well as hazard ratio for this comparison is listed above. Listed below is the number of patients at risk (number of patients alive) per interval, after stratification based on IRF6 expression; in the first interval, number at risk is number of patients alive; in each subsequent interval, number at risk is the number at risk less those who have expired or are censored. 
